\title{
Higher Frequency Band Beamforming Scheme for High Speed Train
}

\author{
Ayotunde O. Laiyemo, Petri Luoto, Pekka Pirinen, and Matti Latva-aho, \\ Centre for Wireless Communications, University of Oulu, P.O. Box 4500, 90014 \\ University of Oulu, Finland. \\ (e-mail: forename.surname@oulu.fi)
}

\begin{abstract}
With the increasing popularity of high speed trains (HSTs) and the traffic forecast for future cellular networks, the need to provide very high data rates using higher frequency bands (HFBs) for train passengers is becoming crucial. In this paper, we present a timer-based beamforming selection algorithm for HST, which exploits the prior knowledge of the position and direction of the HST. A sequential and hierarchical codebook is designed based on the array response vectors and linked to the line-of-sight (LOS) angle-of-arrival/departure base station (BS) -HST link. The effect of velocity feedback errors on the throughput performance was analyzed. The antenna deactivation and the sub-array approaches were considered to mitigate the effect of velocity feedback errors. Evaluation of our proposed beamforming scheme indicates a close performance to the optimal singular value decomposition (SVD) scheme when no velocity feedback error occurs and with the occurrence of velocity feedback errors, the sub-array approach proved to be an efficient way to reduce the effect of the errors.
\end{abstract}

\section{INTRODUCTION}

$\mathbf{P}$ ROVIDING high data rates to onboard users in public transport such as the high speed train (HST) is becoming very crucial. Following recent trends, the data traffic from wireless and mobile devices will continue to experience exponential growth [1] and a larger percentage of mobile users will be onboard users as the deployment of HST in many parts of the world is rapidly growing. Different transmission schemes [2] and techniques [3] have been considered to meet the rapid increase in high data rate demand on HSTs. However, the schemes are limited by the scarce available bandwidth at microwave frequency bands. Recently, the use of higher frequency band (HFB) has been considered for the 5th generation $(5 \mathrm{G})$ wireless communications, since the large available bandwidth at HFBs has been seen as potential to meet the very high and increasing data rate demand [4]. However, due to the sensitivity to the atmosphere and severe propagation loss experienced at these frequency bands, long range transmission is challenging. On the other hand, with the small wavelength and recent advances in modular antenna array technology for HFBs, high beamforming gains can be obtained to combat large propagation loss and therefore are potentially suitable for outdoor communication.

The limitation of microwave bands for future railway was highlighted in [5] and the use of HFBs was suggested for future $5 \mathrm{G}$ communication system for railway (5G-R). In [6], a hybrid spatial modulation beamforming scheme was proposed at HFBs under the HST scenario, where a combination of spatial modulation and hybrid beamforming is used to enhance rate performance. However, channel state information feedback delay was not taken into account, which can significantly affect the rate performance. A modification to the IEEE 802.11ad beam sweeping approach was examined in [7], where the number of beams and an optimum repetition time to sweep through the beams was determined from the velocity estimate of the HST. Also HFB beam switching support for HSTs was considered in [8], in which the beam switching approach leverage on the knowledge of the train position in optimizing the beamwidth to achieve a higher rate. However, information of the selected beam at the BS is required at the HST and beam design details were not examined.

In this paper, we consider a two-hop network architecture, where the communication link is divided into a BS-HST link and a HST-onboard link. We focus on the BS-HST link, since the BS-HST link is the main bottleneck in the whole communication link [9]. The HST consists of moving relay nodes (MRNs) with antenna arrays on the roof top and we assume LOS propagation, since the propagation characteristics of most railway environments exhibit strong LOS component with few scatterers. Also, with the existing cell size, remote radio heads (RRHs) can be used to ensure the presence of LOS propagation as proposed in [10].

We propose a simplified beamforming selection approach based on the characteristics of the railway environment and the HFB channel such that the geometry of the rail, the prior knowledge of the position and velocity of the train are exploited in the design of the algorithm. We show that the proposed algorithm with perfect velocity feedback can achieve a close performance to the optimal SVD scheme. The impact of velocity feedback error on the throughput performance is analyzed and methods to mitigate the impact of velocity feedback errors are examined.

The rest of this paper is organized as follows. In Section II, the system model for the HST network is introduced. The proposed time-based beamforming selection scheme, as applied to the HST, is presented in Section III with the problem formulation. In Section IV, the simulation results are discussed and the conclusion is provided in Section V.

\section{SYSTEM MODEL}

We consider the BS-to-HST link as shown in Fig. 1. The train has multiple carriages, each equipped with a single MRN. The number of MRNs is denoted by $M$. The BS is equipped with $N_{t}$ transmit antennas with $N_{r f}$ RF chains. Each MRN 


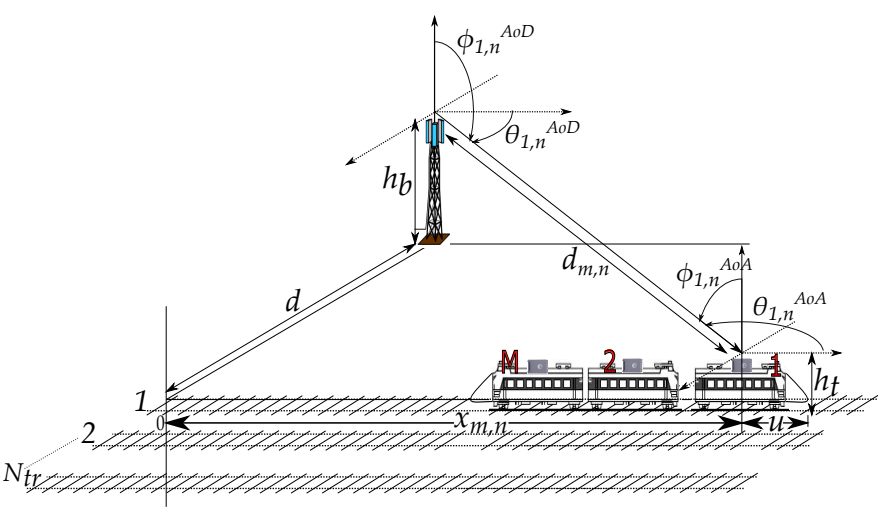

Fig. 1: HST scenario with angle of arrival based beamforming.

is equipped with an external antenna array having $N_{r}$ receive antennas. Note that all the MRNs may not necessarily be active which can be due to limited number of RF chains and number of BSs simultaneously connected to the HST [2].

Let $\mathbf{p}_{m, n}=\left[x_{m, n}, y_{m, j}, h_{t}\right]^{T} \in \mathbb{R}^{3}$ and $\mathbf{q}=\left[0,0, h_{b}\right]^{T} \in$ $\mathbb{R}^{3}$ be locations of the $m^{\text {th }}$ MRN on the HST and the BS. The downlink received signal vector for a single $\mathrm{MRN} \mathbf{y}_{c, i} \in \mathbb{C}^{N_{r}}$ at the $c^{\text {th }}$ sub-carrier for the $i^{\text {th }} \mathrm{RF}$ chain is given as

$$
\mathbf{y}_{c, i}=\mathbf{H}_{c, i} \mathbf{m}_{n, i} \mathbf{s}_{n, i}+\mathbf{n}
$$

where $\mathbf{H}_{c, i} \in \mathbb{C}^{N_{r} \times N_{t_{i}}}$ is the channel matrix of the $i^{\text {th }} \mathrm{RF}$ chain between the serving BS and the MRN. The beamforming weight is given as $\mathbf{m}_{n, i} \in \mathbb{C}^{N_{t_{i}}}$, where the subscript $n$ is the index from a beamforming set $\mathcal{M}$ and $\mathrm{s}_{n, i} \in \mathbb{C}$ denotes the corresponding data symbol for the $i^{\text {th }} \mathrm{RF}$ chain. The additive complex white Gaussian noise vector is defined as $\mathbf{n} \sim \mathcal{C N}\left(0, N_{0} \mathbf{I}_{N_{r}}\right)$ with zero mean and $N_{0}$ variance. The RF chains are implemented such that the channel can be expressed as $\mathbf{H}_{c}=\left[\mathbf{H}_{c, 1}, \ldots, \mathbf{H}_{c, N_{r f}}\right]$. Hence, the received signal to noise ratio (SNR) at the $c^{\text {th }}$ sub-carrier can be written as

$$
\Gamma_{c, i}=\frac{\left|\mathbf{w}_{n}^{H} \mathbf{H}_{c} \mathbf{m}_{n}\right|^{2}}{\left\|\mathbf{w}_{n}\right\|^{2} N_{0}}
$$

where $\mathbf{w}_{n} \in \mathbb{C}^{N_{r}}$ denotes the receive filter for the MRN.

\section{TIMER-BASED BEAMFORMING FOR HST COMMUNICATION}

\section{A. Problem formulation}

In order to maximize the received SNR, a joint trans$\mathrm{mit} /$ receive beamforming is proposed, i.e.,

$$
\begin{array}{cl}
\underset{\left\{\mathbf{m}_{n}, \mathbf{w}_{n}\right\}}{\operatorname{maximize}} & \Gamma_{c} \\
\text { subject to } & \mathbf{m}_{n} \in \mathcal{M} \\
& \mathbf{w}_{n} \in \mathcal{W}
\end{array}
$$

where $\mathcal{M}$ and $\mathcal{W}$ are the sets of transmit and receive vectors.

With channel knowledge at the BS and HST, the optimum beamformers can be solved by singular value decomposition (SVD) if $N_{t}=N_{r f}$. However, in a high mobility scenario, full knowledge of the channel is not feasible. With no knowledge of the channel, the optimal weights can be solved by an exhaustive search through the sets $\mathcal{M}$ and $\mathcal{W}$. But in a real-time
TABLE I: Relationship between $B_{g}$ and $B_{w}$.

\begin{tabular}{|c|c|c|c|c|}
\hline Size of antenna array $(N)$ & 8 & 16 & 32 & 64 \\
\hline Beam gain $\left(B_{g}\right)$ & 8 & 16 & 32 & 64 \\
\hline Beamwidth $\left(B_{w}\right)$ & 0.2225 & 0.11125 & 0.0556 & 0.0278 \\
\hline
\end{tabular}

sensitive scenario like the HST, the selected weights will be outdated due to feedback delay. Taking into account the characteristics of the railway environment and the directionality of HFB propagation, the AoA and AoD of the LOS propagation path can be estimated based on prior knowledge of the HST position and velocity. Hence, the optimal beamforming weight $\mathbf{m}_{n}$ and receive filter $\mathbf{w}_{n}$ can be aligned with the estimated AoA and AoD such that the steering vectors are expressed as

$$
\begin{gathered}
\mathbf{m}_{n}=\mathbf{a}_{t}\left(\theta_{l_{\max }}^{A o D}, \phi_{l_{\max }}^{A o D}\right) \\
\mathbf{w}_{n}=\mathbf{a}_{r}\left(\theta_{l_{\max }}^{A o A}, \phi_{l_{\max }^{A o D}}^{A \text { max }}\right) .
\end{gathered}
$$

\section{B. Relationship between array gain and beamwidth}

For HFB channels, the channel matrix can be mainly characterized by the transmit and receive array response vector such that

$$
\mathbf{H}_{c}=\sqrt{\frac{N_{t} N_{r}}{L}} \sum_{l=1}^{L} \alpha_{l} \mathbf{a}_{r}\left(\theta_{l}^{A o A}, \phi_{l}^{A o A}\right) \mathbf{a}_{t}\left(\theta_{l}^{A o D}, \phi_{l}^{A o D}\right)^{H}
$$

where $\alpha_{l}$ is the amplitude of the channel gain of the $l^{\text {th }}$ multipath component. Symbols $\mathbf{a}_{r}($.$) and \mathbf{a}_{t}($.$) are the receive$ and transmit array response vectors respectively, while $\theta_{l}^{A o D}$ and $\theta_{l}^{A o A}$ represent the angle of departure and the angle of arrival of the $l^{\text {th }}$ multipath component, respectively. The array response vector can be expressed as

$$
\mathbf{a}(\theta)=\left[1, e^{j(k \Delta \sin (\theta)+\delta)}, \ldots, e^{j((N-1) k \Delta \sin (\theta)+\delta)}\right]^{T}
$$

where $k=2 \pi / \lambda, \lambda$ is the carrier wavelength, $\Delta$ is the antenna spacing and $\delta$ is the phase shift from element to element. For the HST scenario, the far field conditions are assumed to be met, since $N_{t} . \Delta_{t} . \lambda \ll d_{m, n}$ and $N_{r} . \Delta_{r} . \lambda \ll d_{m, n}$ can easily be satisfied. Therefore, the array response vector can form beams in the direction of the phase shift $\delta$ with the beam gain expressed as

$B_{g}=\sum \mathbf{a}(\theta)=e^{j\left(\frac{N-1}{2} \psi\right)} \frac{\sin \left(\frac{N}{2} \psi\right)}{\sin (\psi / 2)}$, with $\psi=k \Delta \sin (\theta)+\delta$.

Assuming omnidirectional antenna elements, the maximum array gain is $N$ and the beamwidth is $1.78 / N$ centering at the steering angle, which is achieved when $\psi=0$ (see proof in Appendix). The array gain is beneficial when there is a beam alignment between the transmit and receive antenna arrays. The direction of the beam can be electronically controlled by phase shifters to follow the movement of the HST with $\delta=-k \Delta \sin \left(\theta_{0}\right)$, where $\theta_{0}$ is the steering angle. The beam alignment problem can be solved if the steering angle is always aligned with the angle of arrival. It is intuitive that increasing the number of antenna elements in the array will lead to increased array gain. However, an increase in array gain will lead to narrower beamwidth making the beam alignment problem more challenging, as reflected in Table I. 


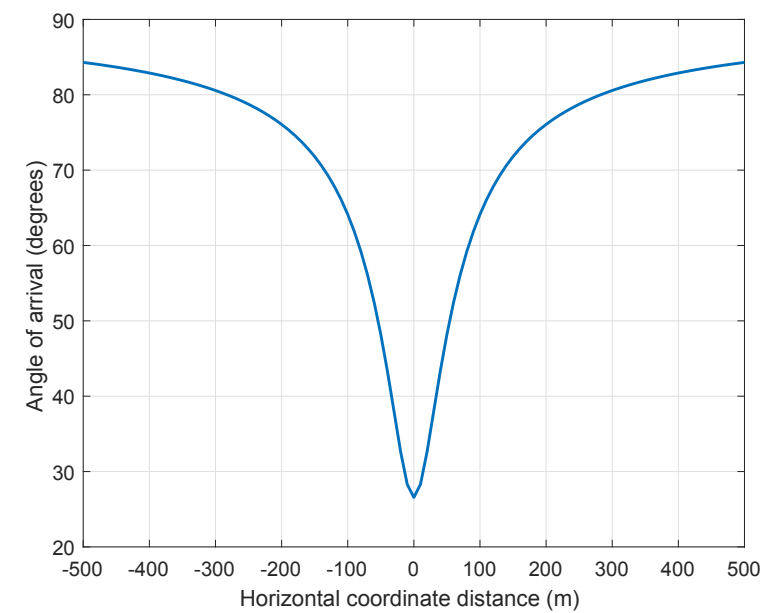

Fig. 2: Rate of change of angle of arrival w.r.t. BS-HST separation distance.

\section{Impact of BS-HST link distance on the angle of arrival}

For the steering angle to be aligned with the angle of arrival, while still maintaining high array gain, precise and real-time main lobe alignment between the BS and the HST is required, as the HST moves at high speed. Knowledge of the rate of change of the angle of arrival w.r.t. the horizontal coordinate distance $x_{m, n}$ and the geometry of the track is required to help achieve a compromise between array gain and beamwidth. Following the propagation scenario D2 in [11], where the minimum perpendicular distance between the track and the BS $d$ is $50 \mathrm{~m}$ with a BS height $h_{b}$ and MRN height $h_{t}$ of $30 \mathrm{~m}$ and $5 \mathrm{~m}$ respectively, the rate of change of angle of arrival is obtained as shown in Fig. 2. We assume a straight line track with BSs in the intervals of $1000 \mathrm{~m}$. Fig. 2 shows how the angle of arrival changes with increasing distance between the BS and the HST. The change in angle of arrival varies rapidly when the HST is close to the BS within the range of approximately $200 \mathrm{~m}$. As the separation distance increases beyond $200 \mathrm{~m}$, the rate of change of angle of arrival reduces. This implies that with prior knowledge of the location and velocity of the HST, electronic steering of the main-lobe with high array gain can be achieved with precision when the HST is farther away from the BS and more challenging at close range. To solve the beam alignment problem at short range, the beamwidth can be widened to accommodate the rapid change in AoA. One way to widen the beamwidth is the use of the deactivation approach, where some antenna elements within the antenna array are deactivated. However, with per-antenna power constraint the maximum total power will be limited. An alternative to the deactivation approach is to split the large antenna array into sub-arrays with $N_{t_{s}}$ antennas in each sub-array. The sub-arrays can be implemented such that the channel can be expressed as $\mathbf{H}=\sqrt{\frac{N_{t} N_{r}}{L}} \sum_{l=1}^{L} \alpha_{l} \mathbf{a}_{r}^{S}\left(\mathbf{a}_{t}^{S}\right)^{H}$ with the array response vector defined as $\mathbf{a}^{S}=\operatorname{vec}\left(\left[\mathbf{a}^{1 S}\left(\theta_{1^{*}, 1}^{A o A}\right), \ldots, \mathbf{a}^{S S}\left(\theta_{1^{*}, S}^{A o A}\right)\right]\right)^{T}$, where the angles used for the sub-arrays are carefully selected to widen the beamwidth by a factor of $S^{2}$. (see proof in Appendix)

\section{Impact of velocity estimation error on the angle of arrival}

In practice, the prior knowledge of the HST's position and velocity might slightly deviate from the real time position and velocity due to environmental factors such as the wind's direction and wind's energy. This deviation can sometimes be critical and lead to beam misalignment. Following the geometry in Fig. 1, we can obtain the estimated train position $\hat{\mathbf{p}}_{m, n}$ at any time instance in terms of the velocity information, i.e., $\left\|\hat{\mathbf{p}}_{m, n}\right\|=\hat{v}\left(\tau_{n}-\tau_{n-1}\right)+\left\|\mathbf{p}_{m, n-1}\right\|$. We assume the deviation in the known velocity $g_{e}$ of the HST follows a normal distribution with mean $\mu$ and variance $\sigma^{2}$ such that the $\hat{v}=v+g_{e}$. The deviation in the AoA as a result of $\hat{v}$ is given as

$$
\theta_{\text {error }}=\tan ^{-1}\left(\frac{\Delta x_{m, n}}{d+\Delta d}\right)
$$

where $\Delta d$ denotes the relative displacement along the perpendicular distance between the BS and the HST, which can occur as a result of wind power. $\Delta x_{m, n}=\left|\hat{x}_{m, n}-x_{m, n}\right|$ is the relative horizontal distance displacement caused by the error in the velocity of the HST. The relative displacement $\Delta d$ is assumed negligible due to the large distance $d$. Hence, the outage probability will depend on the relative displacement $\Delta x_{m, n}$ and the size of the beamwidth $B_{w}$. Therefore, the outage probability is defined as

$$
P_{\text {out }}=\operatorname{Prob}\left\{\theta_{\text {error }}>\alpha B_{w}\right\} \text {. }
$$

Here, the outage probability is defined as the event that the angle of misalignment is larger than a fraction $\alpha$ of the beamwidth of the antenna array.

\section{E. HST beamforming selection scheme}

The beam selection is based on anticipated AoD and AoA of the dominant path, which is calculated from the prior information of the rail environment. This information includes the perpendicular BS-to-track distance $d$, the geometry of the track, the height of the BS $h_{b}$, the height of the HST $h_{t}$, the speed $v$ and initial position of the HST $\mathbf{p}_{m, n}$. Based on these, the angular domain can be determined by casting a normal three dimensional positioning calculation to a single dimension. Given the problem formulation in (3), the sets of transmit $\mathcal{M}$ and receive $\mathcal{W}$ beamforming vectors, which form the codebook, are generated by evenly sampling the angular domain in a sequential order with a total interval of $N_{b}$ on a given track. With the incorporation of the sub-array approach discussed in Section III-C, the codebook is designed to consist of $S$ levels corresponding to the number of subarrays. Each level contains beamforming vectors with a fixed beamwidth and corresponding array gain. The codebook for the beamforming vectors is defined as

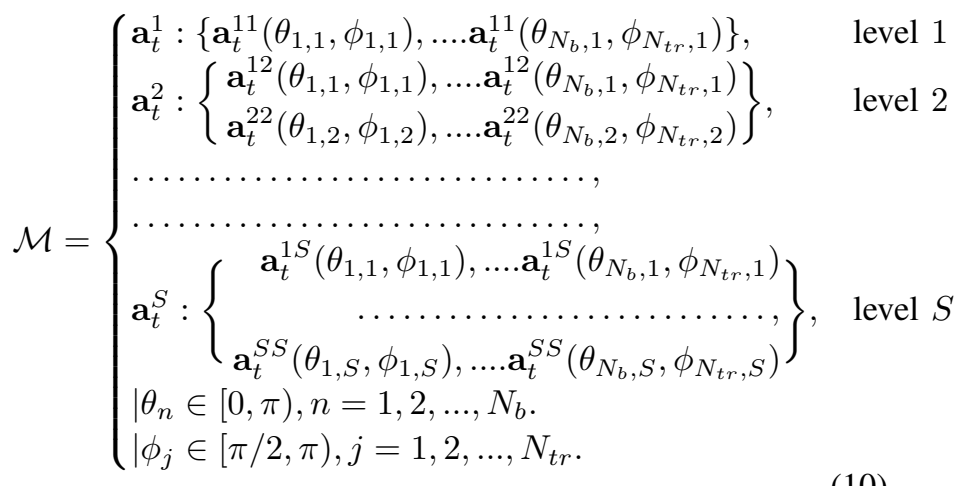


To always ensure beam alignment with the movement of the HST, we propose Algorithm 1, which sequentially selects the beamforming weights from $\mathcal{M}$ at the end of a countdown-timer $(C D T)$. The $C D T$ is configured based on the prior knowledge of the initial HST position and velocity. The proposed algorithm is an extension of the approach in [12]. In this algorithm, velocity estimation error and impact of BS-HST link distance are taken into account along with the introduction of a hierarchical codebook.

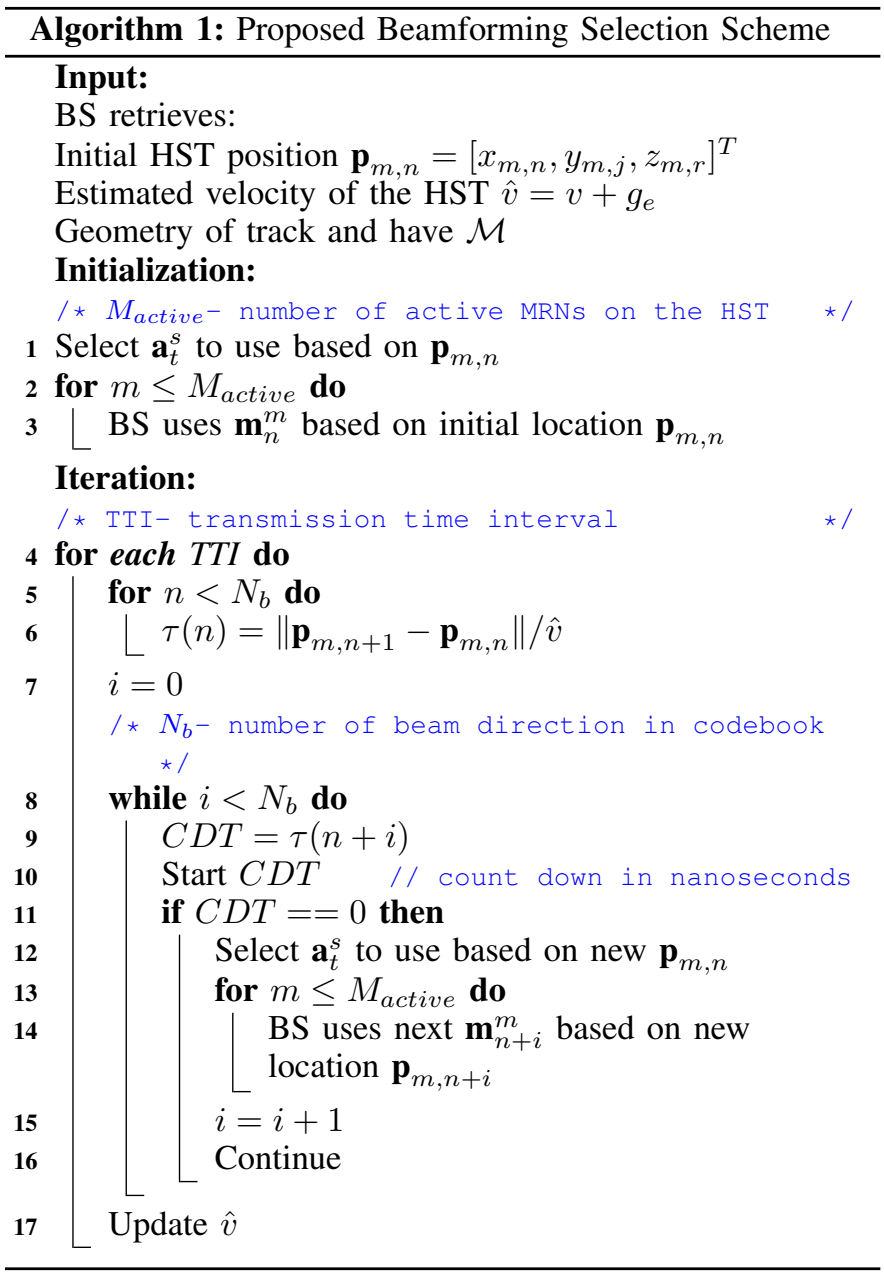

Using Fig. 1 as a reference, $\mathbf{p}_{m, n}=\left[\left(x_{1, n}-2 u(m-\right.\right.$ 1)), $\left.d, h_{t}\right]^{T}$, where $2 u$ is the length of a carriage. The algorithm selects a codebook level $\mathbf{a}_{t}^{s}$ from (10) based on $\mathbf{p}_{m, n}$. The codebook level selection is observed at every transmission time interval (TTI) as the HST position changes. The estimated velocity is also updated at every TTI to minimize the error in the configured CDT. The elapsed time of the CDT triggers the BS to select the next beamforming weights $\mathbf{m}_{n+i}^{m}$ and the process continues until the last beamforming weights $\mathbf{m}_{N_{b}}^{m}$ is selected, which then triggers a handover process to the next BS.

The distance between the BS and the $m^{\text {th }} \mathrm{MRN}$ at the $n^{\text {th }}$ beam can be described as

$$
d_{m, n}=\left\|\mathbf{p}_{m, n}-\mathbf{q}_{m, n}\right\|=\left\{x_{m, n}^{2}+\left(h_{b}-h_{t}\right)^{2}+d^{2}\right\}^{\frac{1}{2}} .
$$

The elevation angle of arrival and departure are same across
TABLE II: Simulation parameters.

\begin{tabular}{|c|c|}
\hline Parameters & Values \\
\hline \hline Carrier frequency & $28 \mathrm{GHz}$ \\
\hline Number of channel realization & 100 \\
\hline BS antenna spacing & 0.5 wavelength \\
\hline Length of each carriage & $30 \mathrm{~m}$ \\
\hline$N_{t} / N_{r}$ & $8,16,32$ \\
\hline Angle of arrival/departure & Uniform distribution $(0, \pi)$ \\
\hline Transmit power $P_{t}$ & $46 \mathrm{dBm}$ \\
\hline
\end{tabular}

the track and are defined as

$$
\begin{aligned}
& \phi_{m, n}^{A o A}=\sin ^{-1}\left(\frac{\left\{d^{2}+x_{m, 1}^{2}\right\}^{\frac{1}{2}}}{d_{m, 1}}\right)=\sin ^{-1}\left(\frac{\left\{d^{2}+x_{m, n}^{2}\right\}^{\frac{1}{2}}}{d_{m, n}}\right) \\
& \phi_{m, n}^{A o D}=\pi-\phi_{m, 1}^{A o A}=\pi-\phi_{m, n}^{A o A} .
\end{aligned}
$$

On the other hand, the azimuth angles are also a function of the horizontal coordinate distance $x_{m, n}$, which varies rapidly due to the speed of the train. The relationship between the azimuth angles and the horizontal coordinate distance can be expressed as

$$
\begin{aligned}
& \theta_{m, n}^{A o A}=\frac{\pi}{2}-\sin ^{-1}\left(\frac{d}{d_{m, n}}\right) \\
& \theta_{m, n}^{A o D}=\pi-\theta_{m, n}^{A o A} .
\end{aligned}
$$

\section{Simulation Results}

In this section, we provide the simulation results to evaluate the performance of the proposed beamforming selection scheme with perfect AoA estimation and compare with the ideal SVD technique. Then we take into account the effect of BS-HST link distance and velocity estimation error in examining the performance of the proposed beamforming selection scheme. We consider a BS with $N_{r f}$ RF chains and $N_{t}$ transmit antennas serving the HST. The number of active MRN is set to 1 . The number of sub-carriers is set to $C=1000$ with a sub-carrier spacing of $500 \mathrm{kHz}$ for 28 $\mathrm{GHz}$ carrier frequency. The channel model used is obtained from a statistical spatial channel model (SSCM) for HFB LOS communication links [13]. It is based on extensive propagation measurements carried out on 28 and $73 \mathrm{GHz}$ band. In each simulation, the performance is averaged over 100 independent channel realizations. The main simulation parameters are listed in Table II.

Fig. 3 shows the data rates achieved for different SNR values with the SVD beamforming scheme and the proposed beamforming selection scheme (PBSS) for multi-stream transmission with $8 \times 8,16 \times 16$ and $32 \times 32 \mathrm{HFB}$ LOS SSCM channel. The result show a significant increase in spectral efficiency as the antenna array size increases from 8 to 32. This is intuitive because as the number of antennas increases, the number of spatial degrees of freedom also increases. However, the number of spatial degrees of freedom is significantly small in relation to the size of the antenna array. This phenomenon is reflected in Fig. 3 where the rate performance of the multistream PBSS shows a close performance to the multi-stream SVD at all antenna configurations despite having a small number of RF chain $N_{r f}$ compared to the size of the antenna array. At $15 \mathrm{~dB}$ SNR, the spectral efficiency gap in Fig. 3 is 


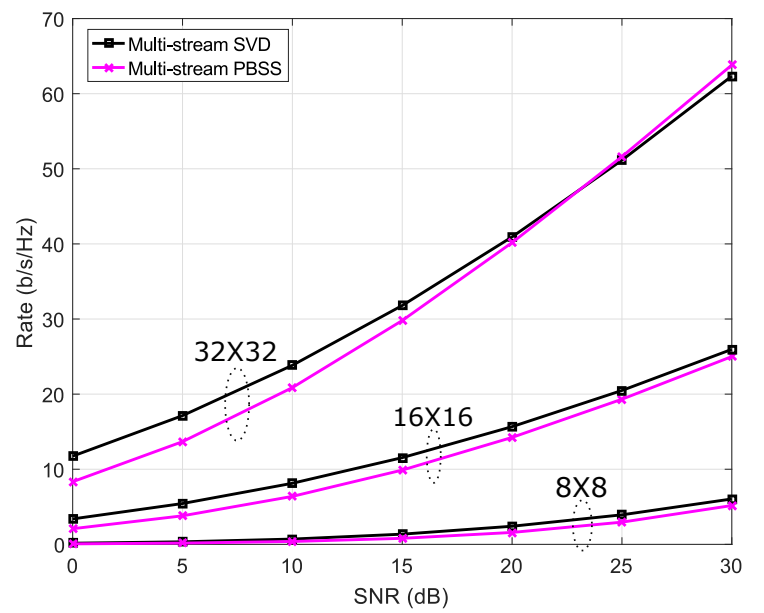

Fig. 3: Performance comparison between SVD and PBSS for different antenna configurations.

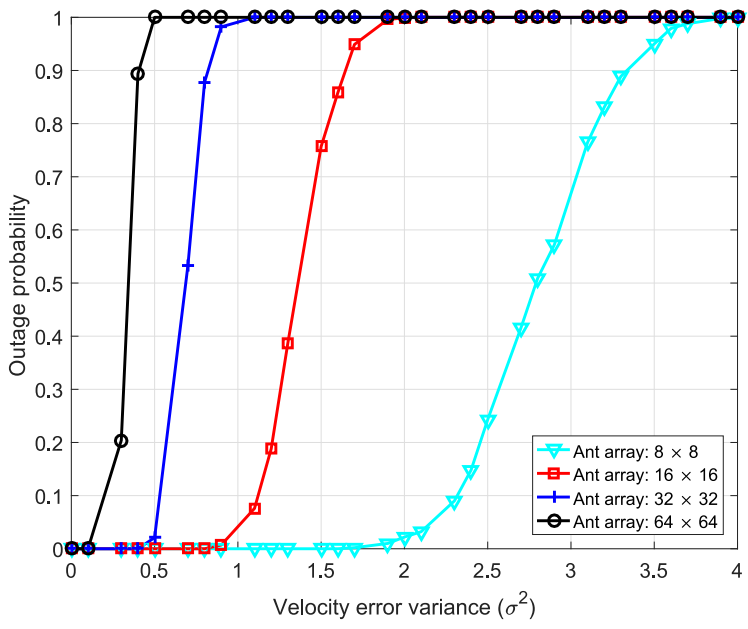

Fig. 4: Outage probability for different antenna configurations.

shown to be $0.56,1.65$ and $2 \mathrm{~b} / \mathrm{s} / \mathrm{Hz}$ for 8,16 and 32 antenna arrays, respectively. Note that $N_{r f}$ was set to 4 for all antenna configurations except for the $32 \times 32$ configuration in which the value was set to 8 .

In Fig. 4, the misalignment of the beams is described by the outage probability as a function of the velocity error variance $\sigma^{2}$. Fig. 4 shows the outage probability for different antenna array size at a horizontal coordinate distance of $x_{m, n}=200$ m and velocity of $v=500 \mathrm{~km} / \mathrm{h}$. The value of $\alpha$ was fixed at 0.05 . The figure shows that with larger antenna arrays, the sensitivity to velocity estimation errors becomes higher leading to an increased chance of beam misalignment. For example, at $\sigma^{2}=2.5$ transmission is in outage approximately $25 \%$ of the time with an array size of 8 and in outage $100 \%$ of the time with an array size of 16, 32 and 64 .

Fig. 5 shows the average rate achieved at different link BS-HST link distances for an antenna array size of 16. In the figure, four different average rates at each specific distance are shown, where "Perfect velocity estimation" and "Imperfect velocity estimation (IVE)" corresponds to the application of Algorithm 1 assuming perfect velocity estimation and applying Algorithm 1 in the presence of velocity error with a variance

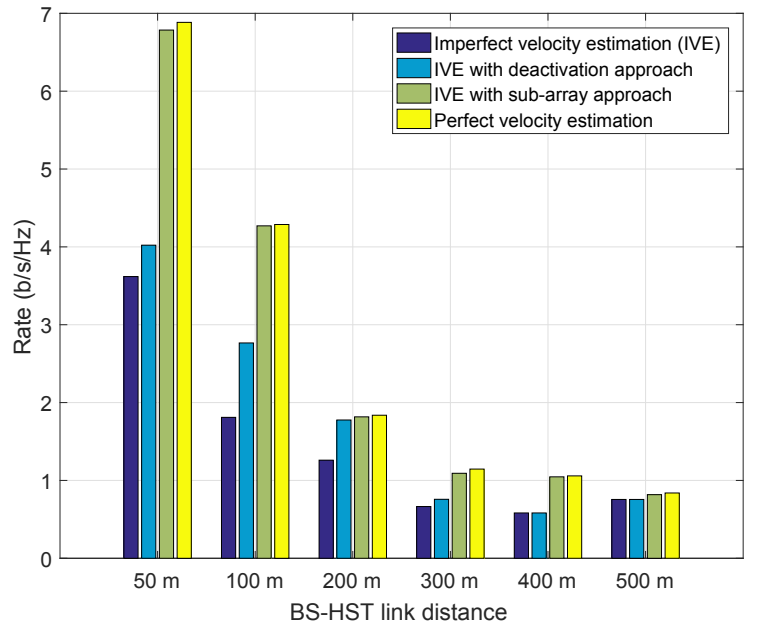

Fig. 5: Average achievable rate at different link BS-HST link distance.

TABLE III: Number of active antennas used for deactivation approach.

\begin{tabular}{|c|c|c|c|c|c|c|}
\hline Link distance $(\mathbf{m})$ & 50 & 100 & 200 & 300 & 400 & 500 \\
\hline Antennas used $\left(A_{u}\right)$ & 4 & 8 & 12 & 12 & 16 & 16 \\
\hline
\end{tabular}

$\sigma^{2}=0.2$, respectively

The impact of velocity feedback error is more significant at shorter link distances. To reduce the impact of velocity feedback error, the deactivation and sub-array approaches are applied with Algorithm 1. The number of sub-arrays used for the sub-array approach is $S=4$, while the number of antennas used for each link distance for the deactivation approach is given in Table III.

The sub-array approach in Fig. 5 shows a close performance to the Perfect velocity estimation since there is a significant reduction in outage probability as a result of the beamwidth expansion by a factor of $S^{2}$. The deactivation approach in Fig. 5 shows a substantial improvement in rate performance compared to Imperfect velocity estimation. However, the deactivation approach provides a worse performance compared to the sub-array approach since the total transmit power is limited by the number of antennas used and the beamwidth is only expanded by a factor of $N_{t} / A_{u}$. Hence, in the deactivation approach the probability for transmission to be in outage is higher than in the sub-array approach.

\section{CONCLUSION}

In this paper, we proposed a timer-based beamforming selection algorithm for HST, which exploits the peculiar characteristics of the railway environment and the velocity feedback of the HST. We developed a sequentially ordered and hierarchical codebook based on the array response vectors and tied to the LOS AoA and AoD between the BS-HST link. We showed that array gains can be achieved with larger antenna arrays, but leads to narrower beamwidth and in turn increases the chance of misalignment between the transmit and receive beams. We also investigated the impact of velocity feedback error with different error variances on the outage probability 
and two approaches were examined in mitigating against the impact of velocity feedback error.

\section{ACKNOWLEDGEMENT}

This work was supported by the Finnish Funding Agency for Technology and Innovation, Nokia, Media Tek, Keysight Technologies, Bittium, and Kyynel.

\section{APPENDIX}

Beam gain $B_{g}$

$$
\begin{aligned}
B_{g}=\sum(\mathbf{a}(\theta)) & =\sum_{n=1}^{N} \exp ^{j(n-1) \psi} \\
\left(\exp ^{j \psi} B_{g}-B_{g}\right) & =\sum_{n=1}^{N} \exp ^{j n \psi}-\sum_{n=1}^{N} \exp ^{j(n-1) \psi} \\
B_{g}=\frac{\exp ^{j N \psi}-1}{\exp ^{j \psi}-1} & =\frac{\exp ^{j(N / 2) \psi}\left(\exp ^{j(N / 2) \psi}-\exp ^{-j(N / 2) \psi}\right)}{\exp ^{j(N / 2) \psi}\left(\exp ^{j(1 / 2) \psi}-\exp ^{-j(1 / 2) \psi}\right)} \\
B_{g} & =\exp ^{j(N-1) \psi / 2} \frac{\sin (N / 2) \psi}{\sin \psi / 2}
\end{aligned}
$$

$\exp ^{j(N-1) \psi / 2}$ accounts for the fact that the physical center of the array is located at $(N-1) \Delta / 2$ and will therefore be neglected. The maximum gain is achieved when $\psi=0$ and following the squeeze theorem we can show that

$\lim _{x \rightarrow 0} \frac{\sin x}{x}=1$, therefore, $\lim _{\psi \rightarrow 0} B_{g}=\lim _{\psi \rightarrow 0} \frac{\sin (N / 2) \psi}{\sin \psi / 2}=N$

\section{Beamwidth $B_{w}$}

If we assume $\psi / 2$ is small, we can apply small angle approximation to $B_{g}$ and normalize to yield

$$
B_{g_{n}}=\frac{1}{N} \frac{\sin (N / 2) \psi}{\psi / 2} .
$$

The beamwidth is obtained when $B_{g_{n}}=0.707$

$$
\begin{aligned}
\frac{\sin (N / 2) \psi}{(N / 2) \psi} & =0.707, \text { this implies }(N / 2) \psi= \pm 1.391 \\
\psi & =k \Delta \sin \theta_{ \pm}+\delta= \pm 2.8 / N \\
\theta_{ \pm} & =\sin ^{-1}\left(\frac{1}{k \Delta}\left(\frac{ \pm 2.8}{N}-\delta\right)\right)
\end{aligned}
$$

With half-wavelength spacing $\Delta=0.5 \lambda$ and element phase shift $\delta=0$, we obtain

$\theta_{ \pm}=\sin ^{-1} \frac{ \pm 0.89}{N}$ and for large value of $N$

small angle approximaion holds yielding

$$
\begin{aligned}
& \theta_{ \pm}=\frac{ \pm 0.89}{N} \text {. Hence, } \\
& B_{w}=\left|\theta_{+}-\theta_{-}\right|=1.78 / N .
\end{aligned}
$$

\section{Beam expansion using the sub-array approach}

Let $S$ be the number of sub-array with $N_{s}$ antenna element in each sub-array. Therefore $B_{g}$ can be rewritten as

$$
\begin{aligned}
B_{g} & =\sum_{s=1}^{S} \sum_{n=1}^{N_{s}} \exp ^{j\left((s-1) N_{s}+(n-1)\right) \psi} \\
& =\sum_{s=1}^{S} \exp ^{j(s-1) N_{s} \psi} \times \sum_{n=1}^{N_{s}} \exp ^{j(n-1) \psi} \\
& =\sum_{s=1}^{S} \exp ^{j(s-1) N_{s} \psi} \times B_{g}^{N_{s}} .
\end{aligned}
$$

The first term is the multiplying factor that achieves maximum value $S$ when $\psi=0$ and $B_{g}^{N_{s}}$ is the beam gain for one subarray. The total beamwidth $B_{w}$ can be seen as the union of the beamwidth of each sub-array $B_{w}^{s}$, i.e., $B_{w}=\cup_{s=1}^{S} B_{g}^{N_{s}}=$ $S \times 1.78 / N_{s}=S^{2} \times 1.78 / N$.

\section{REFERENCES}

[1] "Cisco Visual Networking Index: Forecast and Methodology, 2015 to 2020," White paper, 2016.

[2] A. Laiyemo, H. Pennanen, P. Pirinen, and M. Latva-aho, "Transmission strategies for throughput maximization in high speed train communications: From theoretical study to practical algorithms," IEEE Transactions on Vehicular Technology, vol. 66, no. 4, pp. 2997-3011, 2017.

[3] W. Luo, X. Fang, M. Cheng, and Y. Zhao, "Efficient multiple-group multiple-antenna (MGMA) scheme for high-speed railway viaducts," IEEE Transactions on Vehicular Technology, vol. 62, no. 6, pp. 25582569, 2013.

[4] W. Roh, J.-Y. Seol, J. Park, B. Lee, J. Lee, Y. Kim, J. Cho, K. Cheun, and F. Aryanfar, "Millimeter-wave beamforming as an enabling technology for $5 \mathrm{G}$ cellular communications: theoretical feasibility and prototype results," IEEE Communications Magazine, vol. 52, no. 2, pp. 106-113, 2014.

[5] B. Ai, K. Guan, M. Rupp, T. Kurner, X. Cheng, X.-F. Yin, Q. Wang, G.Y. Ma, Y. Li, L. Xiong et al., "Future railway services-oriented mobile communications network," IEEE Communication Magazine, vol. 53, no. 10, pp. 78-85, 2015.

[6] Y. Cui, X. Fang, and L. Yan, "Hybrid Spatial Modulation Beamforming for mmWave Railway Communication Systems," IEEE Transactions on Vehicular Technology, vol. 65, no. 12, pp. 9597-9606, 2016.

[7] J. Kim and A. F. Molisch, "Enabling Gigabit services for IEEE 802.11 ad-capable high-speed train networks," in Proceedings of IEEE Radio and Wireless Symposium (RWS). IEEE, 2013, pp. 145-147.

[8] V. Va, X. Zhang, and R. W. Heath, "Beam switching for millimeter wave communication to support high speed trains," in Proceedings of 82nd IEEE Vehicular Technology Conference (VTC Fall). IEEE, 2015, pp. $1-5$.

[9] A. O. Laiyemo, H. Pennanen, P. Pirinen, and M. Latva-aho, "Effective deployment of cooperative moving relay nodes in a high speed train," in Wireless Days (WD), 2016. IEEE, 2016, pp. 1-6.

[10] "3rd Generation Partnership Project; Technical Specification Group Radio Access Network; Study on Scenarios and Requirements for Next Generation Access Technologies, 3GPP, Rep. TR 38.913 V14.2.0," Technical Specification, 2016

[11] J. Meinilä, P. Kyösti, T. Jämsä, and L. Hentilä, "WINNER II channel models," Information Society Technologies, 2008.

[12] A. O. Laiyemo, P. Luoto, P. Pirinen, and M. Latva-aho, "Feasibility studies on the use of higher frequency bands and beamforming selection scheme for high speed train communication," Wireless Commun. and Mobile Computing, vol. 2017, 2017.

[13] M. K. Samimi and T. S. Rappaport, "3-D millimeter-wave statistical channel model for $5 \mathrm{G}$ wireless system design," IEEE Transactions on Microwave Theory and Techniques, vol. 64, no. 7, pp. 2207-2225, 2016. 